\title{
Avaliação do vigor de sementes de tomate durante o armazenamento por meio de análise computadorizada de imagens de plântulas
}

\author{
Tomato seed vigor evaluation during storage by seedling \\ computadorized image analysis
}

\author{
Vanessa Neumann Silva ${ }^{1 *}$; Silvio Moure Cicero ${ }^{2}$
}

\section{Resumo}

O objetivo deste trabalho foi verificar a sensibilidade dos parâmetros fornecidos pela análise computadorizada de imagens de plântulas para detectar a redução do vigor de sementes de tomate durante o armazenamento, comparado a testes tradicionalmente utilizados para esta finalidade. Dez lotes de sementes de tomate, cultivar Mariana, foram armazenados por 12 meses em ambiente controlado a $20 \pm 1{ }^{\circ} \mathrm{C}$ e $45-50 \%$ de umidade relativa do ar. O teor de água das sementes foi monitorado e o potencial fisiológico avaliado aos $0,3,6$ e 12 meses após o armazenamento, com os testes de germinação, primeira contagem de germinação, envelhecimento acelerado com solução saturada de sal, emergência de plântulas e com o sistema de análise computadorizada de imagens de plântulas (SVIS). O delineamento experimental utilizado foi inteiramente casualizado em esquema fatorial 4 x 10 (épocas $\mathrm{X}$ lotes) com 4 repetições. Os dados foram submetidos à análise de variância e a comparação de médias por meio do teste de Scott Knott a 5\% de probabilidade. A análise computadorizada de imagens de plântulas de tomate, cinco dias após a semeadura, permite detectar diferenças de vigor entre lotes e a redução do vigor ao longo do armazenamento.

Palavras-chave: Solanum lycopersicum, deterioração de sementes, potencial fisiológico, SVIS

\begin{abstract}
The aim of this study was to determine the sensitivity of the parameters provided by computerized image analysis to detect tomato seed vigor reduction during storage, compared to tests traditionally used for this purpose. Ten lots of tomato seeds, cultivar Mariana, were stored for 12 months in a controlled environment at $20 \pm 1^{\circ} \mathrm{C}$ and $45-50 \%$ relative humidity. The seed moisture content was monitored and physiological potential assessed at $0,3,6$ and 12 months after storage, with germination, first count, accelerated aging with saturated salt solution, seedling emergence tests and with computerized image analyzes of seedlings by SVIS ${ }^{\circledR}$ system. A completely random design was used in a 4 x 10 factorial scheme (4 evaluations periods X 10 lots) with 4 replications. Data were subjected to analysis of variance and mean comparison by the Scott Knott test at $5 \%$ probability. Computer image analysis of tomato seedlings with five days after sowing is able to detect vigor differences between lots and vigor loss during storage.
\end{abstract}

Key words: Solanum lycopersicum, seed deterioration, physiological potential, SVIS

\footnotetext{
${ }^{1}$ Prof A Adjunta, Universidade Federal do Pampa, Campus Itaqui, Itaqui, RS. E-mail: vnpel@yahoo.com.br

${ }^{2}$ Prof. Titular do Dept ${ }^{\circ}$ de Produção Vegetal, Escola Superior de Agricultura 'Luiz de Queiroz", ESALQ, USP, Piracicaba, SP. E-mail: smcicero@usp.br

* Autor para correspondência
} 


\section{Introdução}

$O$ uso de sementes com alto potencial fisiológico é essencial para a obtenção de mudas de hortaliças de alta qualidade. A qualidade de sementes compreende os componentes genético, sanitário, físico e fisiológico, sendo este último definido como a soma da capacidade germinativa e do vigor da semente. A avaliação da qualidade de sementes é fundamental em programas de controle de qualidade de sementes, os quais devem dispor de métodos eficientes, de baixo custo e reproduzíveis. Normalmente esta avaliação é feita por meio do teste de germinação e pode ser complementada por testes de vigor, especialmente porque o teste de germinação por ser conduzido em condições controladas pode superestimar o potencial das sementes.

Os testes de vigor tem sido utilizados principalmente para identificar diferenças associadas ao desempenho de lotes de sementes durante o armazenamento ou após a semeadura, procurando destacar lotes com maior eficiência para o estabelecimento do estande sob ampla variação das condições de ambiente (MARCOS FILHO; KIKUTI; BATISTA, 2009).

Sabe-se que durante o armazenamento, mesmo quando este procedimento é realizado em condições adequadas, as sementes continuam expostas ao processo de deterioração, pois o mesmo é inexorável e irreversível. Portanto, é fundamental dispor de métodos eficientes para verificar o comportamento das sementes durante o período de armazenamento, o que possiblita a escolha adequada de materiais com maior potencial para o estabelecimento de plantas no campo (CANTOS et al., 2011).

Dentre as primeiras manifestações fisiológicas da deterioração de sementes está a redução da velocidade de crescimento de plântulas, que provoca menor velocidade de emergência e que provavelmente esteja associada com a menor capacidade de reorganização das membranas celulares (DELOUCHE; BASKIN, 1973).
Neste contexto, testes que avaliem o crescimento de plântulas constituem-se em alternativas interessantes para avaliar o potencial de conservação das sementes durante o armazenamento; a análise do crescimento de plântulas comumente é realizada com medições manuais de partes das plântulas. Entretanto, este tipo de teste apresenta ao menos duas limitações: os resultados podem variar entre laboratórios por causa dos erros cometidos nas mensurações; requerem tempo excessivo para a aquisição dos resultados. A automatização destas análises pelo uso de processamento computadorizado de imagens pode eliminar essas dificuldades (HOFFMASTER et al., 2003).

Recentemente, para avaliação do vigor de sementes baseado no crescimento de plântulas, Sako et al. (2001) desenvolveram, na Ohio State University/EUA, um sistema automatizado para avaliação do vigor de sementes de alface, denominado Seed Vigor Imaging System (SVIS); para tanto, trabalharam com plântulas escaneadas, cujas partes eram identificadas e marcadas por software específico. Após o processamento das imagens em computador, obtiveram, simultaneamente, dados referentes ao comprimento da raiz primária, do hipocótilo e da plântula toda, além da relação raiz/ hipocótilo e parâmetros baseados na velocidade e uniformidade de crescimento. Este tipo de análise vem possibilitando a determinação do vigor de sementes de outras espécies como milho doce (Zea mays L.) (ALVARENGA; MARCOS FILHO; GOMES JUNIOR, 2012), pepino (Cucumis sativus L.) (CHIQUITO; GOMES JUNIOR; MARCOS FILHO, 2012), crotalária -(Crotalaria juncea L.) (SILVA et al., 2012), soja (Glycine max L.) (MARCOS FILHO; KIKUTI; LIMA, 2009) e melão (Cucumis melo L.) (MARCOS FILHO et al., 2006), além de possibilitar a avaliação do efeito do condicionamento fisiológico em sementes de milho doce (GOMES JUNIOR et al., 2009) e a avaliação do efeito de tratamentos fungicidas na qualidade de sementes de mamona (Ricinus communis L.) (KOBORI et al., 2010) e amendoim (Arachis 
hypogaea L.) (MARCHI; CICERO; GOMES JUNIOR, 2011).

O método consiste na captação de imagens digitais múltiplas, em escaner, de plântulas, que em seguida são processadas em computador, gerando valores numéricos que, coletivamente representam o potencial fisiológico das sementes, com base em parâmetros preestabelecidos. Os dados incluem comprimento de plântulas e índices de vigor, de uniformidade de desenvolvimento e de crescimento de plântulas (SAKO et al., 2001). Após o processamento das imagens, o software gera automaticamente valores numéricos referentes aos índices de vigor, de uniformidade de desenvolvimento e de crescimento (valores de 0 a 1000 , diretamente proporcionais ao vigor).

Não existem pesquisas até o momento sobre o uso desta técnica para a avaliação do vigor de sementes de tomate durante o armazenamento, portanto, o objetivo deste trabalho foi verificar a sensibilidade dos parâmetros fornecidos pela análise computadorizada de imagens de plântulas para detectar a redução do vigor de sementes de tomate durante o armazenamento, comparado a testes comumente utilizados para esta finalidade.

\section{Material e Métodos}

A pesquisa foi conduzida nos Laboratórios de Análise de Sementes e de Análise de Imagens do Departamento de Produção Vegetal da Escola Superior de Agricultura "Luiz de Queiroz" da Universidade de São Paulo, em Piracicaba, SP. Sementes de tomate, cultivar Mariana, provenientes de dez lotes, foram acondicionadas em embalagens de papel aluminizado, hermeticamente fechadas, e armazenadas em ambiente controlado a $20 \pm 1^{\circ} \mathrm{C}$ e $45-50 \%$ de umidade relativa do ar. A caracterização inicial das sementes foi realizada por meio dos testes discriminados a seguir.

Teor de água: utilizou-se o método da estufa, a $105 \pm 3{ }^{\circ} \mathrm{C}$, por 24 horas, com duas amostras de 1,0g para cada lote e os resultados foram expressos em porcentagem (base úmida) (BRASIL, 2009). Germinação e primeira contagem de germinação: quatro repetições de 50 sementes para cada lote foram distribuídas sobre duas folhas de papel mata-borrão umedecidas com quantidade de água equivalente a 2,5 vezes a massa seca do substrato, no interior de caixas plásticas do tipo "gerbox" $(11 \times 11 \times 3,5 \mathrm{~cm})$ e em seguida foram colocadas em uma câmara do tipo BOD, regulada a temperatura de 20-30 ${ }^{\circ} \mathrm{C}$ alternada, com 8 horas de luz e 16 horas de escuro. As avaliações foram realizadas aos 5 e 14 dias após a semeadura (BRASIL, 2009). Os resultados foram expressos em percentagem de plântulas normais para cada lote. Envelhecimento acelerado com solução saturada de sal (EASS): uma camada de sementes foi distribuída sobre tela metálica acoplada a uma caixa plástica do tipo gerbox $(11 \times 11 \times 3,5 \mathrm{~cm})$ contendo $40 \mathrm{ml}$ de solução saturada de $\mathrm{NaCl}$ no fundo, em substituição à água (JIANHUA; McDONALD, 1996). Cada caixa foi tampada e colocadas em uma câmara do tipo BOD, regulada a temperatura de $41{ }^{\circ} \mathrm{C}$, por um período de 48 horas; em seguida foi determinado o teor de água das sementes e realizado teste de germinação com quatro repetições de 50 sementes para cada lote, sendo as plântulas avaliadas após cinco dias (MARCOS FILHO, 1999).

Emergência de plântulas: quatro repetições de 50 sementes por lote foram semeadas em bandejas de isopor contendo substrato comercial próprio para produção de mudas de olerícolas $\left(\right.$ Plantmax $\left.^{\circledR}\right)$. Aos 14 dias após a semeadura foi realizada a contagem de plântulas emersas (RODO; TILLMANN; VILLELA, 1998).

Análise computadorizada de imagens de plântulas por meio do software SVIS: quatro repetições de 25 sementes de cada lote foram distribuídas em rolos de papel toalha umedecidos com água destilada na quantidade de 2,5 vezes a massa do papel e expostas a temperatuara de 20$30{ }^{\circ} \mathrm{C}$ alternada em germinador durante 4 dias na ausência de luz. Em seguida as plântulas foram 
transferidas do papel toalha para uma folha de cartolina com coloração preta, sendo as imagens das plântulas obtidas por meio do uso de escaner modelo HP Scanjet 2410, montado de maneira invertida no interior de uma caixa de alumínio, operado por software Photosmart, com resolução de 100 dpi. Em seguida as imagens foram analisadas com o uso do software SVIS (Seed Vigor Imaging System) instalado em computador Pentium IV, operado por sistema Windows XP Profissional. Nessa análise, o eixo raiz/hipocótilo de cada plântula foi marcado, pelo próprio programa, em vermelho. Após a análise e a avaliação de cada plântula, o software SVIS forneceu os valores para comprimento de plântulas (CP) e índices de vigor (IV) e de crescimento de plântulas (IC). Os dados de comprimento de plântulas foram fornecidos pelo programa em pixels e após a tabulação dos mesmos foi realizada transformação para $\mathrm{cm}$, considerandose que um pixel corresponde a $0,0254 \mathrm{~cm}$.

Foram realizadas quatro épocas de avaliação, aos 0, 3, 6 e 12 meses após o armazenamento. $\mathrm{O}$ delineamento experimental utilizado foi inteiramente casualizado em esquema fatorial $4 \times 10$ (épocas x lotes) com quatro repetições. Os dados foram submetidos à análise de variância e comparação de médias por meio do teste de Scott Knott a 5\% de significância.

\section{Resultados e Discussão}

De acordo com os resultados indicados na Tabela 1, é possível observar que o teor de água das sementes dos dez lotes foi mais alto no início da pesquisa, em torno de $9 \%$, e praticamente não diferiu entre os lotes, e nas demais épocas de análise ficou entre 7,3 a 8\%, também não havendo grande diferença entre os lotes, o que é aceitável, pois as sementes foram acondicionadas em embalagens de papel aluminizado, hermeticamente fechados, e armazenadas em ambiente com controle de temperatura e umidade relativa do ar. É importante mencionar esses resultados, pois é fundamental que não ocorram diferenças consideráveis no teor de água das sementes dos diferentes lotes, considerando-se que aquelas com maior teor de água tem maior probabilidade de germinarem antes, comprometendo os resultados dos testes de vigor que levam em conta o crescimento de plântulas.

Tabela 1. Valores médios do teor de água (TA) de dez lotes de sementes de tomate, cultivar Mariana, armazenadas em ambiente controlado, em Piracicaba, São Paulo, aos 0 (E1), 3 (E2), 6 (E3) e 12 (E4) meses de armazenamento.

\begin{tabular}{ccccc}
\hline \multirow{2}{*}{ LOTE } & \multicolumn{4}{c}{ TA (\%) } \\
\cline { 2 - 5 } & E1 & E2 & E3 & E4 \\
\hline 1 & 9,0 & 7,9 & 8,0 & 7,9 \\
2 & 9,1 & 7,8 & 7,9 & 7,5 \\
3 & 9,6 & 7,9 & 7,9 & 7,4 \\
4 & 9,6 & 7,8 & 7,8 & 7,3 \\
5 & 9,5 & 7,7 & 7,6 & 7,3 \\
6 & 9,0 & 7,6 & 7,7 & 7,6 \\
7 & 9,1 & 7,6 & 7,8 & 7,4 \\
8 & 8,9 & 7,5 & 7,6 & 7,5 \\
9 & 9,5 & 7,8 & 7,9 & 7,8 \\
10 & 9,4 & 7,5 & 7,6 & 7,7 \\
\hline
\end{tabular}

Fonte: Elaboração dos autores.

$\mathrm{Na}$ avaliação inicial dos lotes (época 1) todos os parâmetros utilizados detectaram diferenças de germinação e vigor entre os lotes. A caracterização dos lotes de maior e menor vigor, contudo, variou em função do parâmetro analisado, já que os testes utilizados baseiam-se em princípios diferentes. No percentual de germinação os lotes 2, 4, 5, 6, 7 e 10 foram superiores aos demais, mas nos demais testes estes mesmos lotes apresentaram comportamento diferente, como por exemplo, os lotes 5 e 10 tiveram baixo desempenho na primeira contagem de germinação e no envelhecimento acelerado, sendo portanto, fundamental o uso de vários parâmetros de vigor para avaliar a qualidade de sementes com maior confiabilidade.

A análise de imagens de plântulas via SVIS na primeira época revelou diferenças entre os lotes 
somente baseada no parâmetro comprimento de plântulas (CP). É interessante observar que os lotes 3,9 e 10 , que tiveram menor comprimento, tiveram menor velocidade de crescimento, avaliada pela primeira contagem de germinação, assim como menor desempenho no envelhecimento acelerado. De maneira similar a este resultado, trabalhando com sementes de soja (Glycine max L.), Marcos Filho, Kikuti e Lima (2009) verificaram que o SVIS ${ }^{\circledR}$ é suficientemente sensível para avaliação do vigor de sementes desta espécie, com eficiência comparável à do teste de envelhecimento acelerado.

Os demais parâmetros da análise de imagens (índices de vigor e de crescimento de plântulas) não apontaram diferenças entre os lotes na primeira época de avaliação, contudo, este resultado não se repetiu nas demais épocas; é possível que na caracterização inicial dos lotes, devido às pequenas diferenças no crescimento inicial das plântulas, para a maioria dos lotes, os índices SVIS não tenham detectado diferenças entre lotes.

Nas épocas seguintes, todos os testes utilizados foram eficientes para separar os lotes quanto ao vigor. Os índices de vigor e de crescimento forneceram resultados semelhantes, indicando os lotes 1 e 2 como de maior potencial na segunda e terceira épocas e os lotes 1, 4 e 8 na quarta época; estes resultados concordam parcialmente com aqueles obtidos na primeira contagem de germinação e no envelhecimento acelerado; entretanto, vale ressaltar que o comprimento de plântulas e os índices de vigor estratificaram os lotes em 4 níveis de vigor aos 3 meses de armazenamento (época 2), enquanto que o envelhecimento acelerado não foi capaz de mostrar diferenças entre os lotes neste mesmo período de análise e a primeira contagem de germinação, assim como a emergência de plântulas, separaram os lotes em apenas 2 grupos.

No entanto, tanto os resultados obtidos na análise de imagens como nos demais testes de vigor não indicaram redução de qualidade das sementes na segunda época, comparado à caracterização inicial, para a maioria dos lotes. Como as sementes estavam armazenadas em local com condições ambientais controladas, o avanço da deterioração ocorreu em menor velocidade. Aos 6 meses de armazenamento (época 3), foi possível observar redução do vigor por meio do comprimento de plântulas e dos índices de vigor e de crescimento de plântulas, obtidos na análise de imagens via SVIS, para a maioria dos lotes (Tabela 4), o que não ocorreu para os testes de primeira contagem de germinação, de envelhecimento acelerado e de emergência de plântulas (Tabelas 2 e 3). Resultados satisfatórios tem sido relatados na literatura com o uso da análise de imagens de plântulas para avaliação do vigor de sementes de várias espécies, como pepino (Cucumis sativus L.) (CHIQUITO; GOMES JUNIOR; MARCOS FILHO, 2012), crotalária (Crotalaria juncea L.) (SILVA et al., 2012), trigo (Triticum aestivum L.) (SILVA; GOMES JUNIOR; CICERO, 2012), milho doce (Zea mays L.) (ALVARENGA; MARCOS FILHO; GOMES JUNIOR, 2012) e melão (Cucumis melo L.) (MARCOS FILHO et al., 2006). 
Tabela 2. Valores médios de plântulas normais obtidas no teste de germinação $(\mathrm{G})$ e no teste de primeira contagem de germinação (PC), de dez lotes de sementes de tomate, cultivar Mariana, armazenadas em ambiente controlado, em Piracicaba, São Paulo, aos 0 (E1), 3 (E2), 6 (E3) e 12 (E4) meses.

\begin{tabular}{|c|c|c|c|c|}
\hline \multirow{2}{*}{ LOTE } & \multicolumn{4}{|c|}{ G (\%) } \\
\hline & E1 & E2 & E3 & E4 \\
\hline 1 & $77 \mathrm{bB}^{*}$ & $90 \mathrm{aA}$ & $93 \mathrm{aA}$ & $45 \mathrm{cC}$ \\
\hline 2 & $86 \mathrm{Aa}$ & $89 \mathrm{aA}$ & $93 \mathrm{aA}$ & $53 \mathrm{bB}$ \\
\hline 3 & $78 \mathrm{bB}$ & $88 \mathrm{aA}$ & $91 \mathrm{aA}$ & $37 \mathrm{dC}$ \\
\hline 4 & $83 \mathrm{aB}$ & $86 \mathrm{aA}$ & $94 \mathrm{aA}$ & $42 \mathrm{cC}$ \\
\hline 5 & $81 \mathrm{aB}$ & $84 \mathrm{aB}$ & $95 \mathrm{aA}$ & $47 \mathrm{cC}$ \\
\hline 6 & $86 \mathrm{aB}$ & $82 \mathrm{bB}$ & $93 \mathrm{aA}$ & $45 \mathrm{cC}$ \\
\hline 7 & $83 \mathrm{aB}$ & $85 \mathrm{aB}$ & $90 \mathrm{aA}$ & $70 \mathrm{aC}$ \\
\hline 8 & $74 \mathrm{bC}$ & $92 \mathrm{aA}$ & $81 \mathrm{bB}$ & $55 \mathrm{bD}$ \\
\hline 9 & $72 \mathrm{bB}$ & $74 \mathrm{bB}$ & $85 \mathrm{bA}$ & $43 \mathrm{cC}$ \\
\hline 10 & $79 \mathrm{aA}$ & $77 \mathrm{bA}$ & $78 \mathrm{bA}$ & $61 \mathrm{bB}$ \\
\hline $\mathrm{CV}(\%)$ & 9.6 & 9.6 & 9.6 & 9.6 \\
\hline \multirow{2}{*}{ LOTE } & \multicolumn{4}{|c|}{ PC $(\%)$} \\
\hline & E1 & E2 & E3 & E4 \\
\hline 1 & $76 \mathrm{aA}$ & $81 \mathrm{aA}$ & $90 \mathrm{aA}$ & $41 \mathrm{cB}$ \\
\hline 2 & $82 \mathrm{aA}$ & $82 \mathrm{aA}$ & $91 \mathrm{aA}$ & $57 \mathrm{bB}$ \\
\hline 3 & $69 \mathrm{bB}$ & $57 \mathrm{bB}$ & $87 \mathrm{aA}$ & $56 \mathrm{bC}$ \\
\hline 4 & $76 \mathrm{aA}$ & $66 \mathrm{bB}$ & $90 \mathrm{aA}$ & $56 \mathrm{bC}$ \\
\hline 5 & $70 \mathrm{bB}$ & $64 \mathrm{bB}$ & $93 \mathrm{aA}$ & $37 \mathrm{cC}$ \\
\hline 6 & $81 \mathrm{aA}$ & $60 \mathrm{bB}$ & $88 \mathrm{aA}$ & $47 \mathrm{cC}$ \\
\hline 7 & $82 \mathrm{aA}$ & $84 \mathrm{aA}$ & $85 \mathrm{bA}$ & $65 \mathrm{aB}$ \\
\hline 8 & $67 \mathrm{bC}$ & $88 \mathrm{aA}$ & $72 \mathrm{bB}$ & $66 \mathrm{aC}$ \\
\hline 9 & $62 \mathrm{bC}$ & $72 \mathrm{aB}$ & $80 \mathrm{bA}$ & $45 \mathrm{cD}$ \\
\hline 10 & $69 \mathrm{bB}$ & $76 \mathrm{aA}$ & $63 \mathrm{bB}$ & $53 \mathrm{bC}$ \\
\hline $\mathrm{CV}(\%)$ & 15 & 15 & 15 & 15 \\
\hline
\end{tabular}

*Médias seguidas por mesma letra minúscula na coluna e maiúscula na linha não diferem pelo teste de Scott Knott a 5\% de significância.

Fonte: Elaboração dos autores.
Nos testes de germinação, primeira contagem de germinação e envelhecimento acelerado, o principal tipo de anormalidade de plântulas verificado foi o encurtamento e engrossamento de raízes, o que é contabilizado na análise de imagens, porém, esta característica não é analisada na emergência de plântulas, o que pode ter contribuído para este teste ter menos sensibilidade na separação dos lotes em níveis de vigor. Contudo, embora a primeira contagem de germinação e o envelhecimento acelerado tenham possibilitado a identificação de diferenças de vigor entre os lotes em cada época, não foram eficientes na detecção da perda do vigor durante o armazenamento, para a maioria dos lotes.

Assim como nesta pesquisa, para sementes de amendoim (Arachis hypogaea L.) Marchi, Cicero e Gomes Junior (2011) verificaram que a análise de imagens de plântulas via SVIS além de apresentar maior rapidez na obtenção dos resultados, mostrou de forma mais evidente a redução do potencial fisiológico no decorrer dos períodos de armazenamento, quando comparados aos testes de envelhecimento acelerado e de emergência de plântulas em campo, ou seja, a redução do potencial fisiológico foi detectada antecipadamente pela análise computadorizada de imagens de plântulas. 
Tabela 3. Resultado de envelhecimento acelerado com solução salina saturada (EASS) e emergência de plântulas (EP), de dez lotes de sementes de tomate, cultivar Mariana, armazenadas em ambiente controlado, em Piracicaba, São Paulo, aos 0 (E1), 3 (E2), 6 (E3) e 12 (E4) meses.

\begin{tabular}{|c|c|c|c|c|}
\hline \multirow{2}{*}{ LOTE } & \multicolumn{4}{|c|}{ EASS (\%) } \\
\hline & E1 & E2 & E3 & E4 \\
\hline 1 & $73 \mathrm{bA}^{*}$ & $66 \mathrm{aA}$ & $67 \mathrm{bA}$ & $43 \mathrm{aB}$ \\
\hline 2 & $79 \mathrm{aA}$ & $72 \mathrm{aA}$ & $81 \mathrm{aA}$ & $32 \mathrm{bB}$ \\
\hline 3 & $69 \mathrm{bB}$ & $67 \mathrm{aB}$ & $88 \mathrm{aA}$ & $34 \mathrm{bC}$ \\
\hline 4 & $71 \mathrm{bA}$ & $75 \mathrm{aA}$ & $76 \mathrm{bA}$ & $27 \mathrm{bB}$ \\
\hline 5 & $74 \mathrm{bA}$ & $67 \mathrm{aB}$ & $80 \mathrm{aA}$ & $32 \mathrm{bC}$ \\
\hline 6 & $66 \mathrm{bB}$ & $62 \mathrm{aB}$ & $83 \mathrm{aA}$ & $21 \mathrm{bC}$ \\
\hline 7 & $85 \mathrm{aA}$ & $72 \mathrm{aB}$ & $85 \mathrm{aA}$ & $48 \mathrm{aC}$ \\
\hline 8 & $85 \mathrm{aA}$ & $75 \mathrm{aB}$ & $66 \mathrm{bB}$ & $51 \mathrm{aC}$ \\
\hline 9 & $63 \mathrm{bA}$ & $55 \mathrm{aB}$ & $41 \mathrm{cC}$ & $52 \mathrm{aB}$ \\
\hline 10 & $63 \mathrm{bA}$ & $68 \mathrm{aA}$ & $41 \mathrm{cB}$ & $61 \mathrm{aA}$ \\
\hline \multicolumn{5}{|l|}{$\mathrm{CV}(\%)$} \\
\hline \multirow{2}{*}{ LOTE } & \multicolumn{4}{|c|}{ EP (\%) } \\
\hline & E1 & E2 & E3 & E4 \\
\hline 1 & $90 \mathrm{aA}$ & $93 \mathrm{aA}$ & $90 \mathrm{aA}$ & $94 \mathrm{aA}$ \\
\hline 2 & $93 \mathrm{aA}$ & $89 \mathrm{aA}$ & $76 \mathrm{bB}$ & $97 \mathrm{aA}$ \\
\hline 3 & $92 \mathrm{aA}$ & $89 \mathrm{aA}$ & $81 \mathrm{aA}$ & $93 \mathrm{aA}$ \\
\hline 4 & $81 \mathrm{bB}$ & $92 \mathrm{aA}$ & $90 \mathrm{aA}$ & $92 \mathrm{aA}$ \\
\hline 5 & $87 \mathrm{aA}$ & $88 \mathrm{aA}$ & $90 \mathrm{aA}$ & $94 \mathrm{aA}$ \\
\hline 6 & $81 \mathrm{bB}$ & $84 \mathrm{bB}$ & $91 \mathrm{aA}$ & $93 \mathrm{aA}$ \\
\hline 7 & $82 \mathrm{bB}$ & $91 \mathrm{aA}$ & $94 \mathrm{aA}$ & $95 \mathrm{aA}$ \\
\hline 8 & $88 \mathrm{aA}$ & $92 \mathrm{aA}$ & $93 \mathrm{aA}$ & $91 \mathrm{aA}$ \\
\hline 9 & $75 \mathrm{bB}$ & $77 \mathrm{bA}$ & $89 \mathrm{aA}$ & $84 \mathrm{bA}$ \\
\hline 10 & $78 \mathrm{bB}$ & $88 \mathrm{aA}$ & $64 \mathrm{cC}$ & $92 \mathrm{aA}$ \\
\hline
\end{tabular}

*Médias seguidas por mesma letra minúscula na coluna e maiúscula na linha não diferem pelo teste de Scott Knott a 5\% de significância.

Fonte: Elaboração dos autores.
Comparando-se os resultados de germinação e de vigor ao longo do experimento, é possível observar que o teste de germinação somente permitiu identificar a perda de qualidade das sementes aos 12 meses de armazenamento (época 4), quando os percentuais de germinação foram muito inferiores ao mínimo recomendado pela legislação para atender os padrões de qualidade para comercialização de sementes de tomate no Brasil, que é no mínimo $80 \%$.

Conforme destacado anteriormente, as informações obtidas por meio da análise de imagens de plântulas permitiram detectar a redução do vigor das sementes aos 6 meses de armazenamento, para a maioria dos lotes, o que não foi possível com os testes de primeira contagem de germinação, envelhecimento acelerado e emergência de plântulas (Tabelas 2 a 4). Trabalhando com sementes de trigo (Triticum aestivum L.), Silva, Gomes Junior e Cicero (2012) verificaram redução do vigor de sementes durante o armazenamento quando utilizaram a análise computadorizada de imagens de plântulas e, assim como na presente pesquisa, esta constatação não foi possível por meio de testes tradicionalmente utilizados para a avaliação do vigor de sementes. 
Tabela 4. Valores médios de índice de vigor (IV), índice de crescimento de plântulas (IC) e comprimento de plântulas $(\mathrm{CP})$, obtidos por meio de análise de imagens de plântulas com SVIS, de dez lotes de sementes de tomate, cultivar Mariana, armazenadas em ambiente controlado, em Piracicaba, São Paulo, aos 0 (E1), 3 (E2), 6 (E3) e 12 (E4) meses.

\begin{tabular}{|c|c|c|c|c|}
\hline \multirow{2}{*}{ LOTES } & \multicolumn{4}{|c|}{ IV } \\
\hline & E1 & E2 & E3 & E4 \\
\hline 1 & $638 \mathrm{aB}^{*}$ & $719 \mathrm{bA}$ & $534 \mathrm{bC}$ & $436 \mathrm{aD}$ \\
\hline 2 & $630 \mathrm{aB}$ & $783 \mathrm{aA}$ & $594 \mathrm{aB}$ & $391 \mathrm{bC}$ \\
\hline 3 & $646 \mathrm{aA}$ & $645 \mathrm{cA}$ & $485 \mathrm{cB}$ & $362 \mathrm{bC}$ \\
\hline 4 & $649 \mathrm{aA}$ & $606 \mathrm{dA}$ & $531 \mathrm{bB}$ & $433 \mathrm{aC}$ \\
\hline 5 & $627 \mathrm{aA}$ & $595 \mathrm{dA}$ & $522 \mathrm{bB}$ & $365 \mathrm{bC}$ \\
\hline 6 & $633 \mathrm{aA}$ & $600 \mathrm{dA}$ & $549 \mathrm{bB}$ & $366 \mathrm{bC}$ \\
\hline 7 & $632 \mathrm{aA}$ & $650 \mathrm{cA}$ & $519 \mathrm{bB}$ & $385 \mathrm{bC}$ \\
\hline 8 & $631 \mathrm{aA}$ & $612 \mathrm{dA}$ & $476 \mathrm{cB}$ & $410 \mathrm{aC}$ \\
\hline 9 & $605 \mathrm{aA}$ & $584 \mathrm{dA}$ & $516 \mathrm{bB}$ & $378 \mathrm{bC}$ \\
\hline 10 & $607 \mathrm{aA}$ & $577 \mathrm{dA}$ & $522 \mathrm{bB}$ & $327 \mathrm{bC}$ \\
\hline $\mathrm{CV}(\%)$ & 6.31 & 6.31 & 6.31 & 6.31 \\
\hline \multirow{2}{*}{ LOTES } & \multicolumn{4}{|c|}{$\mathrm{IC}$} \\
\hline & E1 & E2 & E3 & E4 \\
\hline 1 & $539 \mathrm{aB}$ & $672 \mathrm{bA}$ & $411 \mathrm{cC}$ & $262 \mathrm{aD}$ \\
\hline 2 & $576 \mathrm{aB}$ & $737 \mathrm{aA}$ & $495 \mathrm{aC}$ & $231 \mathrm{aD}$ \\
\hline 3 & $569 \mathrm{aA}$ & $557 \mathrm{cA}$ & $350 \mathrm{cB}$ & $178 \mathrm{bC}$ \\
\hline 4 & $585 \mathrm{aA}$ & $507 \mathrm{~dB}$ & $388 \mathrm{cC}$ & $281 \mathrm{aD}$ \\
\hline 5 & $524 \mathrm{aA}$ & $480 \mathrm{dA}$ & $381 \mathrm{cB}$ & $183 \mathrm{bC}$ \\
\hline 6 & $560 \mathrm{aA}$ & $481 \mathrm{~dB}$ & $427 \mathrm{bB}$ & $159 \mathrm{bC}$ \\
\hline 7 & $569 \mathrm{aA}$ & $557 \mathrm{cA}$ & $403 \mathrm{cB}$ & $205 \mathrm{bC}$ \\
\hline 8 & $542 \mathrm{aA}$ & $499 \mathrm{dA}$ & $370 \mathrm{cB}$ & $226 \mathrm{aC}$ \\
\hline 9 & $564 \mathrm{aA}$ & $484 \mathrm{~dB}$ & $443 \mathrm{bB}$ & $209 \mathrm{bC}$ \\
\hline 10 & $527 \mathrm{aA}$ & $475 \mathrm{~dB}$ & $431 \mathrm{bB}$ & $145 \mathrm{bC}$ \\
\hline $\mathrm{CV}(\%)$ & 9.93 & 9.93 & 9.93 & 9.93 \\
\hline \multirow{2}{*}{ LOTES } & \multicolumn{4}{|c|}{$\mathrm{CP}(\mathrm{CM})$} \\
\hline & E1 & E2 & E3 & $\mathrm{E} 4$ \\
\hline 1 & $4.8 \mathrm{aA}$ & $5.5 \mathrm{bA}$ & $3.8 \mathrm{bB}$ & $2.6 \mathrm{aC}$ \\
\hline 2 & $4.6 \mathrm{aB}$ & $6.9 \mathrm{aA}$ & $4.7 \mathrm{aB}$ & $1.9 \mathrm{bC}$ \\
\hline 3 & $4.2 \mathrm{bA}$ & $4.8 \mathrm{cA}$ & $3.1 \mathrm{bB}$ & $1.6 \mathrm{bC}$ \\
\hline 4 & $4.1 \mathrm{bA}$ & $4.1 \mathrm{dA}$ & $3.7 \mathrm{bA}$ & $2.2 \mathrm{aB}$ \\
\hline 5 & $4.6 \mathrm{aA}$ & $4.3 \mathrm{dA}$ & $3.7 \mathrm{bB}$ & $1.6 \mathrm{bC}$ \\
\hline 6 & $4.6 \mathrm{aA}$ & $4.5 \mathrm{cA}$ & $4.2 \mathrm{aA}$ & $1.6 \mathrm{bB}$ \\
\hline 7 & $4.3 \mathrm{bB}$ & $5.2 \mathrm{cA}$ & $4.0 \mathrm{aB}$ & $2.4 \mathrm{aC}$ \\
\hline 8 & $4.6 \mathrm{aA}$ & $4.7 \mathrm{cA}$ & $4.2 \mathrm{aA}$ & $2.0 \mathrm{aB}$ \\
\hline 9 & $3.9 \mathrm{bA}$ & $4.0 \mathrm{dA}$ & $3.6 \mathrm{bA}$ & $1.6 \mathrm{bB}$ \\
\hline 10 & $4.0 \mathrm{bA}$ & $4.0 \mathrm{dA}$ & $3.9 \mathrm{bA}$ & $1.1 \mathrm{bB}$ \\
\hline $\mathrm{CV}(\%)$ & 12.73 & 12.73 & 12.73 & 12.73 \\
\hline
\end{tabular}

* Médias seguidas por mesma letra minúscula na coluna e maiúscula na linha não diferem pelo teste de Scott Knott a 5\% de significância.

Fonte: Elaboração dos autores.
$\mathrm{Na}$ Figura 1 está ilustrado um exemplo de análise de imagens de plântulas de tomate obtidas na primeira e quarta épocas do experimento; é possível observar clara diferença no comprimento de plântulas entre a época 1 (Figura 1A) e a época 4 (Figura 1B).

Figura 1. Exemplo de análise de imagens de plântulas de tomate no software SVIS, aos 0 (A) e 12 (B) meses de armazenamento, lote 8 , cultivar Mariana, mostrando valores de índice de vigor (vigor index), índice de crescimento de plântulas (Growth) e comprimento de plântulas (Individual measurements).

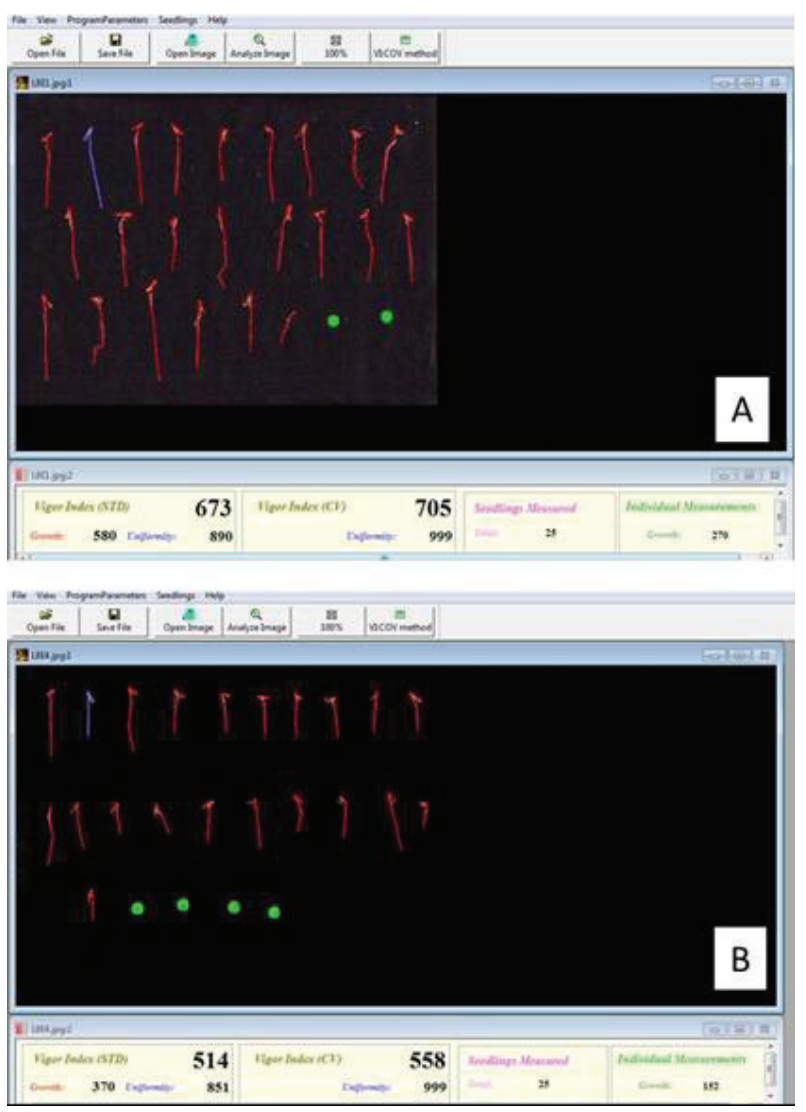

Fonte: Elaboração dos autores.

O uso de parâmetros que permitam a detecção da perda de vigor ao longo do armazenamento é muito importante na análise de sementes, pois possibilita o descarte de materiais com baixo potencial para a emergência de plantas. 
Para sementes de hortaliças, como o tomate, a desuniformidade de emergência de plântulas afeta negativamente a produção de mudas, causando prejuízos ao produtor. As perdas neste caso podem ser tanto diretas, pela perda de material propagativo, como indiretas, pois com o atraso na emergência das plântulas, as sementes ficam mais tempo suscetíveis ao ataque de patógenos de solo, podendo ainda atuarem como fonte de inóculo no sistema produtivo, levando a contaminação das demais plantas.

\section{Conclusão}

A análise computadorizada de imagens de plântulas de tomate, com cinco dias após a semeadura, permite detectar diferenças de vigor entre lotes e a redução do vigor ao longo do armazenamento.

\section{Agradecimentos}

À empresa Sakata Seed Sudamérica pelo fornecimento de sementes para a realização desta pesquisa e à Fundação de Amparo à Pesquisa do Estado de São Paulo (FAPESP - Processo 2009/51689-3) pela bolsa de Doutorado concedida à primeira autora e pelo financiamento para realização desta pesquisa.

\section{Referências}

ALVARENGA, R. A.; MARCOS FILHO, J.; GOMES JUNIOR, F. G. Avaliação do vigor de sementes de milho superdoce por meio da análise computadorizada de imagens de plântulas. Revista Brasileira de Sementes, Londrina, v. 34, n. 3, p. 488-494, 2012.

BRASIL. Ministério da Agricultura, Pecuária e Abastecimento. Regras para análise de sementes. Brasília: SNDA/DNDV/CLAV, 2009. 395 p.

CANTOS, A. A.; TUNES, L. M.; BARBIERI, A. P. P.; TAVARES, L. C. Avaliação de testes de vigor em sementes de aveia branca (Avena sativa L.). Revista da FZVA, Uruguaiana, v. 18, n. 2, p. 1-11, 2011.
CHIQUITO, A. A.; GOMES JUNIOR, F. G.; MARCOS FILHO, J. Assessment of physiological potential of cucumber seeds using the software seedling vigor imaging system ${ }^{\circledR} \quad(\mathrm{SVIS} \circledast)$. Revista Brasileira de Sementes, Londrina, v. 34, n. 2, p. 255-263, 2012.

DELOUCHE, J. C.; BASKIN, C. C. Accelerated aging techniques for predicting the relative storability of seed lots. Seed Science and Technology, Zürich, v. 1, n. 2, p. 427-452, 1973.

GOMES JUNIOR, F. G.; MONDO, V. H. V.; CICERO, S. M.; McDONALD, M. B.; BENNETT, M. A. Evaluation of priming effects on sweet corn seeds by SVIS. Seed Technology, Sacramento, v. 31, n. 1, p. 95-100, 2009.

HOFFMASTER, A. L.; FUJIMURA, K.; McDONALD, M. B.; BENNETT, M. A. An automated system for vigor testing three-day-old soybean seedlings. Seed Science and Technology, Zürich, v. 31, n. 3, p. 701-713, 2003.

JIANHUA, Z.; McDONALD, M. B. The saturated salt accelerated aging test for small-seeded crops. Seed Science and Technology, Zürich, v. 25, n. 1, p. 123-131, 1996.

KOBORI, N. N.; MASTRANGELO, T.; CICERO, S. M.; CASSIERI, P.; MORAES, M. H. D.; WALDER, J. M. M. Effects of gamma radiation on physiological and phytosanitary qualities of Brazilian Castor Bean seeds, Ricinus communis (cv. IAC Guarani). Research Journal of Seed Science, New York, v. 3, n. 1, p. 70-81, 2010.

MARCHI, J. L.; CICERO, S. M.; GOMES JUNIOR, F. G. Utilização da análise computadorizada de plântulas na avaliação do potencial fisiológico de sementes de amendoim tratadas com fungicida e inseticida. Revista Brasileira de Sementes, Londrina, v. 33, n. 3, p. 652-662, 2011.

MARCOS FILHO, J. Teste de envelhecimento acelerado In: KRZYZANOWSKI, F. C.; VIEIRA, R. D.; FRANÇA NETO, J. de B. Vigor de sementes: conceitos e testes. Londrina: ABRATES, 1999. p. 1-21.

MARCOS FILHO, J.; BENNETT, M. A.; MCDONALD, M. B.; EVANS, A. F.; GRASSBAUGH, E. M. Assessment of melon seed vigour by an automated computer imaging system compared to traditional procedures. Seed Science and Technology, Zürich, v. 35, n. 2, p. 485-497, 2006.

MARCOS FILHO, J.; KIKUTI, A. L. P.; LIMA, L. B. Métodos para avaliação do vigor de sementes de soja, incluindo a análise computadorizada de imagens. Revista Brasileira de Sementes, Londrina, v. 31, n. 1, p. 102-112, 2009. 
RODO, A. B.; TILLMANN, M. A. A.; VILLELA, F. A. Testes de vigor na avaliação da qualidade fisiológica de sementes de tomate. Revista Brasileira de Sementes, Londrina, v. 20, n. 1, p. 23-28, 1998.

SAKO, Y.; MCDONALD, M. B.; FUJIMURA, K.; EVANS, A. F.; BENNETT, M. A. A system for automated seed vigour assessment. Seed Science and Technology, Zürich, v. 29, n. 3, p. 625-636, 2001.
SILVA, C. B.; LOPES, M. M.; MARCOS FILHO, J.; VIEIRA, R. D. Automated system of seedling image analysis (SVIS) and electrical conductivity to assess sun hemp seed vigor. Revista Brasileira de Sementes, Londrina, v. 34, n. 1, p. 55-60, 2012.

SILVA, V. N.; GOMES JUNIOR, F. G.; CICERO, S. M. Computerized imaging analysis of seedlings for assessment of physiological potential of wheat seeds. Revista Brasileira de Sementes, Londrina, v. 34, n. 4, p. 589-596, 2012. 